\title{
Properties of intermediate band materials
}

\author{
C. Tablero , P. Palacios ，J.J. Fernández , P. Wahnón
}

\begin{abstract}
Intermediate Band Materials (MIB) have been proposed in previous works as a new kind of photovoltaic materials. These materials are principally characterized by an intermediate partially occupied band, isolated from the valence and conduction bands of the host semiconductor. These materials have a theoretical efficiency greater than that of conventional solar cells because they can absorb photons with a lower energy than the bandgap of the original host semiconductor. However, although the operation of these solar cells has been described, it is necessary to use a method to be able to propose the material which has these properties. In this work, a theoretical study of the electronic and optoelectronic properties using quantum-mechanics calculations is presented. The results confirm that MIB are able to absorb photons of lower energy than the host semiconductor.
\end{abstract}

Keywords: Intermediate Band materials; Efficiency; Properties

\section{Introduction}

In previous works, the importance of intermediate band materials in the field of solar energy has been stressed [1]. The operating model of the this solar cell and a 


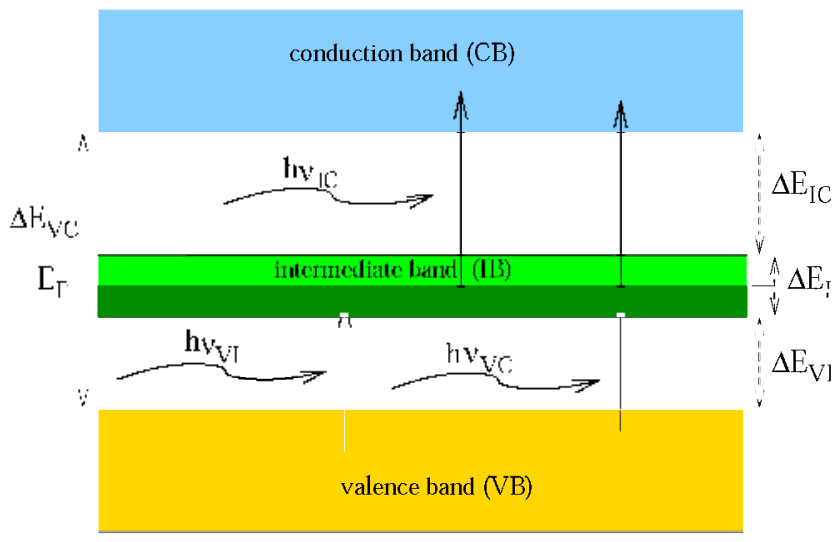

Fig. 1. Model of the Intermediate band solar cell. In the figure $\Delta E_{\mathrm{VI}}, \Delta E_{\mathrm{IC}}$ and $\Delta E_{\mathrm{VC}}$ are the gaps between the VB-IB, IB-CB and VB-CB, respectively, $\Delta E_{i}$ is the band width of the IB and $E_{\mathrm{F}}$ is the Fermi energy. The arrows represent the possible transitions in this material.

diagram of them are shown in Fig. 1. It can be seen in this figure that this MIB can absorb photons with lower energy than the bandgap of the original host semiconductor to promote electrons from the valence band (VB) to the intermediate band (IB), and from this to the conduction band (CB). The conventional process of transition from VB to CB also takes place.

The solar cells based on this MIB have several characteristic shown in Fig. 1. Between them, the presence of IB can be highlighted, isolated from the CB and VB bands. If this requirement is not fulfilled, the electron-phonon interaction can produce thermal relaxation due to the interaction between the electrons and the phonons coming from the lattice. Moreover, the IB must have a finite width to avoid, as much as possible, processes of non-radiative recombination and has to be partially filled to be able to absorb low-energy photons that promote electrons from the VB to the half filled IB and from this one to the CB.

This kind of solar cell presents efficiencies higher than those established by the Shockley limit [2], as has been demonstrated in the previous work of Luque and Marti [1]. They show that the efficiency is increased based on thermodynamic arguments and the operation of this kind the cell. Quantum dots [3] have been proposed to engineer the intermediate band and [4] have synthesized intermediate band materials from II-VI diluted oxides.

Our purpose in this work, is to analyse, from a microscopic level and with quantum-mechanic methodology, some of the proposed materials that have an intermediate band. Moreover, this methodology will be applied to determining the electronic and optical properties.

To achieve this task, we will first describe the approximations and methodology used to obtain the electronic properties. In the second part we show some of the results of optical properties obtained from the electronic properties, using these quantum-mechanic tools. 


\section{Electronic properties}

\subsection{Methodology}

For determining the electronic properties of MIB materials, the Schrodinger equation is solved [5] using the Born-Oppenheimer approximation. In this approach the electronic energy is obtained from a frozen nuclear configuration and it acts as a potential for the nuclear movement.

Other approximation used in solids is the pseudopotential [6] approximation to reproduce the core of the atoms, based on the small contribution of core electrons in the bonds. This approximation has the advantage of reducing the computational cost, because the number of electrons is drastically reduced. Only the valence electrons are considered.

The following step is to use the density functional theory (DFT) that allows us to map the interacting many-body problem onto a one-body problem using the Kohn-Sham (KS) method [7]. In this method attention is focused on the electron density $\rho(\vec{r})$, defined as

$$
\rho(\bar{r})=\sum_{\mu}\left|\psi_{\mu}(\vec{r})\right|^{2},
$$

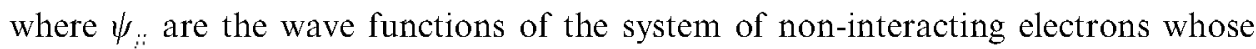
electron density is $\rho$. These orbitals are the solution to the KS equation:

$$
\left(-\frac{1}{2} \nabla^{2}+V_{\mathrm{KS}}[\rho]\right) \psi_{n}=\varepsilon_{n} \psi_{n},
$$

where $V_{\mathrm{KS}}$ is the effective potential of the one electron problem.

$$
V_{\mathrm{KS}}[\rho]-V(r)+\int \frac{\rho\left(\vec{r}^{\prime}\right)}{\left|\vec{r}-\overrightarrow{r^{\prime}}\right|} \mathrm{d} \overrightarrow{r^{\prime}}+V_{x c}[\rho] .
$$

This potential is the sum of the ionic potential acting on the electrons, the average potential of the electron density, the so-called Hartree potential, and the exchangecorrelation potential.

The main problem of this theory is that the exchange-correlation potential is unknown. If this potential were known, the solution would be exact. Unfortunately we must have an approximation. Some of the more extensively used functionals are the local density approximation (LDA) and the generalized gradient approximation (GGA).

To introduce the exchange and correlation term we used the Perdew, Burke and Ernzerhof [8] parameterization for GGA and the Ceperley-Alder [9] parameterization for LDA.

In spite of its approximations, this theory is one of the few that allows the study of large systems such as crystalline solids and allows us to obtain realistic results for many electron systems. However, LDA and GGA approximations have the problem of underestimating the band gaps. Nevertheless, some methods to avoid this inconvenience have been proposed [10]. 
Typically, plane-waves DFT methods have been used to carry out quantum molecular calculations. These methods are very powerful but they require thousands of plane waves to correctly compute the bands. In our case, the KS equations are solved using the SIESTA code [5] with a basis set of localized functions to represent the KS orbitals, in other words, to approximate the KS orbitals as a linear combination of localized functions. In any case, it is convenient that this linear combination satisfies Bloch's theorem when used for solids. With a localized basis set the intermediate band is better described because it is more localized than the conduction band. If the representation of the KS orbitals has translational symmetry, the orbitals will have a new index $\vec{k}$ to indicate a point in the Brillouin zone (BZ). With this choice, the KS orbitals for the solid $\psi_{j \cdot \vec{k}}(\vec{r})$ are built as a linear combination of localized orbitals adapted to the translational symmetry

$$
\psi_{j, k}(r)-|j, k\rangle=\sum_{u} \sum_{n_{u}} C_{\cdot,, n_{u}}^{i, \vec{k}}\left|\vec{k}, u, n_{u}\right\rangle,
$$

where the $j$ index labels the band and $k$ represents a point in the BZ. Each one of these adapted symmetry functions $\left|k, u, n_{u}\right\rangle$ is formed by combinations of functions localized at different atomic positions $\phi_{n_{u}}(r)$ that satisfies Bloch's Theorem

$$
\left|\hat{k}, u, n_{u}\right\rangle=\frac{1}{\sqrt{N}} \sum_{\vec{R}} \mathrm{e}^{\mathrm{i} \vec{k} \vec{R}} \phi_{n_{u}}\left(\dot{r}-\vec{R}-\vec{\tau}_{u}\right),
$$

where $u$ is an index that labels every atom contained in the unit cell of the solid, $n_{u}$ labels every basis function belonging to the atom $u, \tau_{u}$ represents the position of the $u$ th atom within the unit cell, $N$ is the number of cells under consideration, $C_{u, n}^{j, \vec{k}}$ is an expansion coefficient and $R$ is a lattice vector representing the position of each cell within the solid

$$
\dot{R}=n_{1} \vec{a}_{1}+n_{2} \vec{a}_{2}+n_{3} \vec{a}_{3} .
$$

In the last expression $n$ s are integers and the $a_{i} \mathrm{~s}$ are the lattice vectors. The functions $\phi_{i, b_{c}}$ are known as the basis set of the representation for the wave function. Within of the Born-Oppenheimer approximation the total energy, sum of the nuclei energy and the electronic energy of a frozen nuclear configuration, is minimized. The variables implied in this minimization are the coefficients $C_{u, n . .}^{j, k}$.

\subsection{Results}

Using the previous methodology, we have found several different materials that have the intermediate band. These compounds were found by substituting some transition metals in host III-V semiconductors [11]. The resulting structure after the minimization of the total energy is almost cubic as shown in Fig. 2.

The final lattice parameter of the resulting alloy is higher than the original lattice parameter for the host semiconductor and the resulting atomic coordinates are displaced slightly from their original positions. 


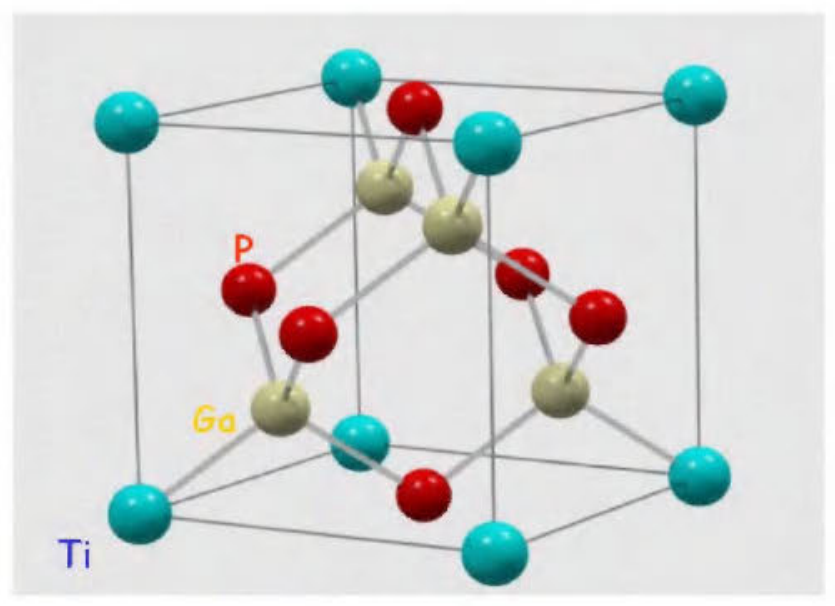

Fig. 2. Crystalline structure of materials proposed.
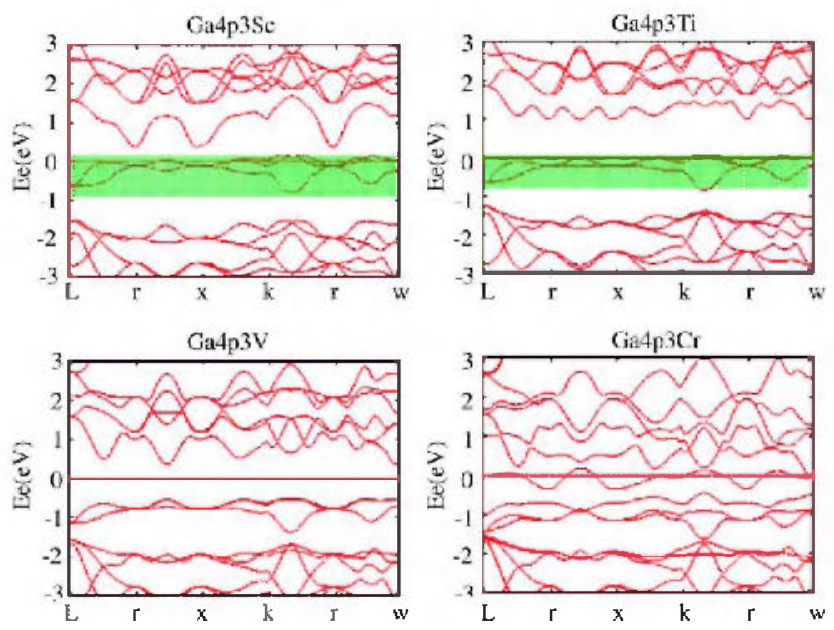

Fig. 3. Band diagrams for some compounds analyzed in several directions in the Brillouin zone.

In Fig. 3 we present some band diagrams for a series of $\mathrm{Ga}_{4} \mathrm{P}_{3} \mathrm{M}$ compounds, where $\mathrm{M}$ represents a transition metal atom. This figure shows a perfectly isolated intermediate band (highlighted in the figure) for the situations where $M$ is $\mathrm{Ti}$ or Sc. The Fermi level in these cases cross the intermediate bands. When $M$ is $V$, the compound is a semiconductor and when it is $\mathrm{Cr}$ we have a metallic material. An analysis of the projected density of states and the electronic density [12] reveals that the intermediate band for $M=\mathrm{Ti}$ and Sc is mainly formed by the $t_{2 \mathrm{~g}}\left(d_{x v}, d_{x z}\right.$ and $d_{v z}$ ) orbitals belonging to the transition metal. 


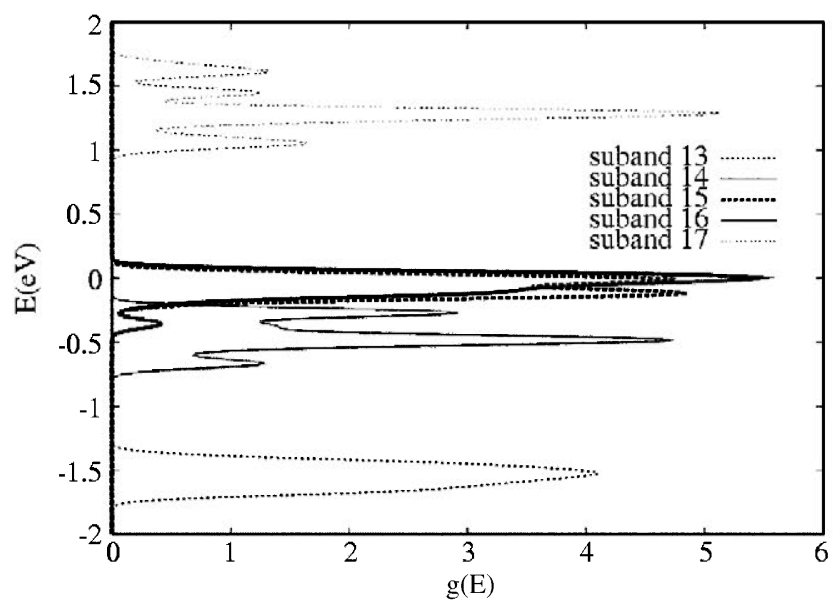

Fig. 4. Contribution to DOS $(g(E))$ of the sub-band 13 (last sub-band of the VB), 14, 15, 16 (sub-bands of the IB) and 17 (first sub-band of the CB).

The contribution to the density of states (DOS) of the last sub-band (labeled as 13) of the VB is represented in Fig. 4, the three sub-bands (labeled in increasing order of energy as 14,15 and 16) form the IB and the first sub-band (labeled as 17) of the CB. Notice that with pseudopotential approximation we consider the valence electrons of the Ti $\left(3 d^{2} 4 s^{2}\right), G a\left(4 s^{2} 4 p^{1}\right)$ and $\mathrm{P}\left(3 s^{2} 3 p^{3}\right)$. Therefore, in the cubic cell shown in Fig. 2 there are 31 electrons. In Fig. 3 these electrons occupy the first 13 sub-bands with 26 electrons and 5 electrons in the IB formed by the 14, 15 and 16 sub-bands.

The origin of energies in Fig. 4 is the Fermi energy. Therefore, the sub-bands below the origin will be full, like is the sub-band 13 of the VB, and above the origin is empty like the first sub-band 17 of the CB. One sub-bands of the IB, the labeled as 14 is full and the 15 and 16 are partially full. This projected DOS in the sub-bands, obtained from electronic properties, will be important when the optical properties are analyzed.

\section{Optical properties}

The optical properties are related to the electronic properties, characterized principally by the energies $\left(E_{b, \vec{k}}\right)$ of the different bands in the $\mathrm{BZ}$ and the corresponding occupation numbers $\left(f_{v, \vec{R}}\right)$, and the matrix element of the momentum operator [13]. For example, the optical conductivity can be calculated using the Kubo formula [14]:

$$
\begin{aligned}
\sigma(\omega)= & \frac{2 \mathrm{e}^{2} \Omega}{m^{2} c n \omega} \sum_{v} \sum_{c} \int_{\mathrm{BZ}} \mathrm{d} \vec{k}|\langle v, \vec{k}|\hat{p}| c, \vec{k}\rangle|^{2} \\
& \times\left[f_{v, \vec{k}}-f_{c, \vec{k}} \delta \delta\left(E_{c, \vec{k}}-E_{v, \vec{k}}-\hbar \omega\right),\right.
\end{aligned}
$$


where $m$ and $e$ are the mass and charge of the electron respectively, $c$ is the speed of light in a vacuum, $n$ is the refraction index of the material, $\Omega$ is the volume of the Brillouin Zone (BZ), $f_{v, \vec{k}}$ are the occupation numbers and $\hbar \omega$ is the photon energy.

From analyses of the projected DOS on the sub-bands shown in Fig. 4, the optical transitions between the sub-bands 13 to 14 do not happen because of the different occupations in the sub-bands. The sub-band 13 and 14 in Fig. 4 is situated below the Fermi energy (zero energy). Thus, these sub-bands are full and the occupation term in the conductivity $\left[f_{v, \vec{k}}-f_{n, \vec{k}}\right]$ is zero. The occupation factor for the transitions between sub-bands 13 to 15 and 13 to 16 is similar because of the projected DOS of the sub-bands 15 and 16 are similar for above the Fermi energy. Of course, the transition 13 to 15 is a little more intense because the DOS for sub-band 16 is a little greater than sub-band 15 . The difference between the maximum of the projected DOS between the sub-bands $13-15$ and $13-16$ is around $1.5-1.6 \mathrm{eV}$. Therefore, the contributions of these transitions to the optical properties, as the absorption coefficient and the optical conductivity, are this energy range. This fact is shown in the Fig. 5, where the contribution of the conductivity from the different sub-bands is represented. In Fig. 5, we have only considered the states at the top the VB (sub-band 13), at the bottom the CB (sub-band 17) and the states in the IB (sub-bands 14-16).

For the transitions between the 13-17 and 14-17 sub-bands, the occupation factors are similar. The 13 and 14 sub-bands are full (below zero in Fig. 4) and 17 is empty (below Fermi energy in Fig. 4). However, these transitions are different with respect to the energy range contribution and the relative intensity contribution to the optical properties. The difference between the energy ranges of the projected DOS in sub-bands 13 and 14 with respect to sub-band $17 \mathrm{can}$ be seen in Fig. 4. Therefore, the contribution to the optical properties of sub-bands 13-17 has greater energies than the 14-17 transition. This fact is confirmed in Fig. 5. Moreover, the contribution of the 14-17 sub-band transition is more intense because of the greater DOS corresponding to sub-band 14. There are more accessible states in sub-band 14 to carry out the transition. This intensity difference is reflected in Fig. 5.

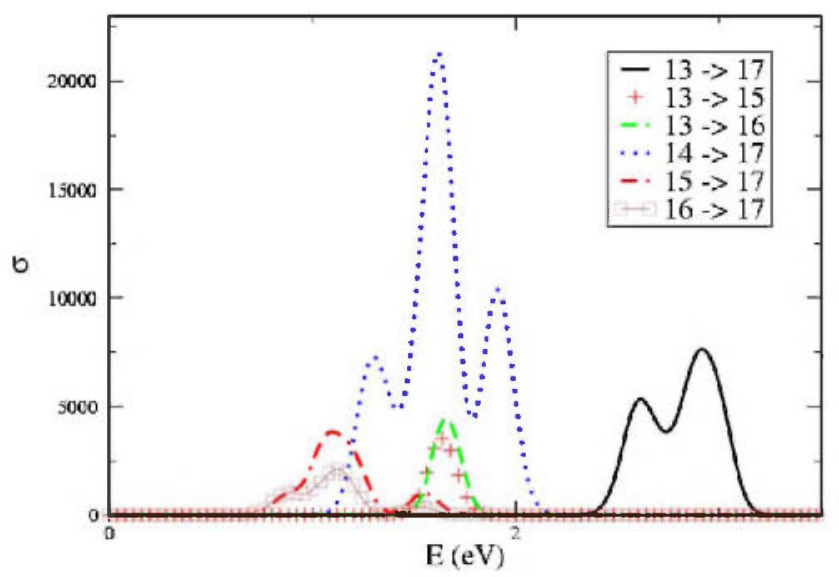

Fig. 5. Contribution to total conductivity $\left(\mathrm{eV} \Omega^{-1}\right)$ for the $\mathrm{Ga}_{4} \mathrm{P}_{3} \mathrm{Ti}$ system of the sub-bands 13 to 17 . 
Moreover, from the previous analyses and in Fig. 5, it can be seen that the energy at which the material begins to absorb photons, corresponding to the transition of the $\mathrm{IB}$ to the conduction band $\mathrm{CB}$, is smaller than the corresponding to the transitions from the VB to $\mathrm{CB}$. In fact, the absorptions of the VB to IB also correspond to a lower energy than the VB to $\mathrm{CB}$. Therefore, this compound is able to absorb lower-energy photons than the traditional VB to $\mathrm{CB}$ to promote electrons from IB to $\mathrm{CB}$ and the VB to IB. For this reason, the total conductivity higher to lower energies than the semiconductor host $(\mathrm{GaP})$.

\section{Conclusions}

We have described and applied some theoretical methods for studying the electronic and optical properties of solids. These application have focused on various materials, some of them exhibiting a partially full intermediate band, of great importance in solar cells. With this methodology, we have obtained the relaxed nuclei positions and the KS orbitals using a basis set of localized functions adapted to the symmetry.

After obtaining the electronic properties, we have calculated some optoelectronic properties, such as the optical conductivity, focussed on the contributions of the principals sub-bands of the VB, IB and CB. For this, we have chosen the $\mathrm{Ga}_{4} \mathrm{P}_{3} \mathrm{Ti}$ system, one the materials, to present an intermediate band.

One of the problems of the methodology used in DFT is that the electronic exchange and correlation terms have to be approximated as has been previously explained. We have developed and implemented [10] new theoretical methodologies in order to substitute the usual LDA and GGA exchange terms in the calculation of the electronic properties. With this new methodology the well known problem of gap underestimation will be avoided.

\section{Acknowledgments}

This work has been supported by Plan Nacional de I+D of Spain (Ref. MAT2002-01076) and by the European Commission through the funding of the project FULLSPECTRUM (Ref. N: SES6-CT-2003-502620).

\section{References}

[1] A. Luque. A. Martí. Phys. Rev. Lett. 78 (1997) 5014.

[2] W. Shockley, H.J. Queisser, J. Appl. Phys. 32 (1961) 510.

[3] A. Marti, L. Cuadra, A. Luque. Proceedings of the 28th IEEE Photovoltaics Specialists Conference, vol. 940, IEEE, New York, 2000;

A. Luque, A. Marti. Progress in Photovoltaics, Research and Applications 9 (2) (2001) 73.

[4] K.M. Yu, W. Walukiewicz, J. Wu, W. Shan, J.W. Beeman, M.A. Scarpulla, O.D. Dubon, P. Becla, Phys. Rev. Lett. 91 (24) (2003) 246403-1; 
K.M. Yu, W. Walukiewicz1, J. Wu, W. Shan, M.A. Scarpulla, O.D. Dubon, J.W. Beeman, P. Becla, Proceedings of the 19th European Photovoltaic Solar Energy Conference, WIP-Renewable Energies and ETA, Munich and Florence, to be published.

[5] J.M. Soler, E. Artacho, J.D. Gale, A. Garcia, J. Junquera, P. Ordejón, D. Sánchez-Portal, J. Phys.: Condens. Matter 14 (2002) 2745.

[6] N. Troullier, J.L. Martins, Phys. Rev. B 43 (1991) 1993.

[7] W. Kohn, L.J. Sham, Phys. Rev. 140 (1965) A1133-A1138.

[8] J.P. Perdew, A. Zunger, Phys. Rev. B 23 (1981) 5048.

[9] D. M Ceperley, B.J. Alder, Phys. Rev. Lett. 45 (1980) 566.

[10] J.J. Fernández, C. Tablero, P. Wahnón, Int. J. Quant. Chem. 91 (2003) 157

J.J. Fernández, C. Tablero. P. Wahnón, Rev. Comp. Mat. Science (2003) 274-286.

[11] P. Wahnón, C. Tablero, Phys. Rev. B 65 (2002) 165115.

[12] C. Tablero, P. Wahnón, App. Phys. Lett. 82 (2003) 151.

[13] C. Tablero, A.J. Garcia, J.J. Fernández, P. Palacios, P. Wahnón, Comp. Mater. Sci. 27 (2003) 58.

[14] K. Ohno, K. Esfarjani, Y. Kawazoe, Computational Materials Science. From ab initio to Monte Carlo Methods, Springer, Berlin, 1999. 